\title{
Numerical Assessment of a Class of High Order Stokes Spectrum Solver
}

\author{
Etienne Ahusborde ${ }^{1, *}$, Mejdi Azaïez ${ }^{2}$ and Ralf Gruber ${ }^{3}$ \\ ${ }^{1}$ Laboratoire de Mathématiques et de leurs Applications de Pau, CNRS / Univ Pau \\ $\mathcal{E}$ Pays Adour, Fédération IPRA, UMR5142, 64000, Pau, France. \\ 2 Bordeaux INP, I2M (UMR CNRS 5295), 33607 Pessac, France. \\ ${ }^{3}$ Chemin du Daillard 11, 1071 Chexbres/VD, Switzerland.
}

Received October 2, 2017; Accepted January 10, 2018

\begin{abstract}
It is well known that the approximation of eigenvalues and associated eigenfunctions of a linear operator under constraint is a difficult problem. One of the difficulties is to propose methods of approximation which satisfy in a stable and accurate way the eigenvalues equations, the constraint one and the boundary conditions. Using any non-stable method leads to the presence of non-physical eigenvalues: a multiple zero one called spurious modes and non-zero one called pollution modes. One way to eliminate these two families is to favor the constraint equations by satisfying it exactly and to verify the equations of the eigenvalues equations in weak ways. To illustrate our contribution in this field we consider in this paper the case of Stokes operator. We describe several methods that produce the correct number of eigenvalues. We numerically prove how these methods are adequate to correctly solve the 2D Stokes eigenvalue problem.
\end{abstract}

AMS subject classifications: 76D07,65N35, 34L16

Key words: Stokes eigenvalues problem, spectral method.

\section{Introduction}

The 2D Stokes eigenvalue problem on a square domain is considered in this paper as model example with a conservation law of the type $\nabla \cdot \boldsymbol{u}=0$. With this test example it is possible to discuss the various numerical problems that appear when flux conservation has to be satisfied in the incompressible Navier-Stokes. If these constraint condition cannot be satisfied precisely, so-called spectral pollution [9] appears and the numerical

${ }^{*}$ Corresponding author. Email addresses: etienne.ahusborde@univ-pau.fr (E. Ahusborde), mejdi.azaiez @enscbp.fr (M. Azaïez), ralf .gruber@bluewin.ch (R. Gruber) 
approach does not stably converge to the physical solution. The reason is that due to regularity constraints imposed by standard numerical approximation methods, the energy cannot reach the minimum required by the physics. In fact, current numerical methods satisfy the boundary conditions strongly, the operator equations and the constraints only weakly.

In Section 2, we propose a non-exhaustive list of methods to deal with the 2D Stokes eigenvalue problem. Specifically, if the constraint $\nabla \cdot \boldsymbol{u}=0$ is satisfied by a $\boldsymbol{u}=\nabla \times \psi$ ansatz, the number of degrees of freedom remains the same as in the unconstrained Laplacian problem. As a consequence, besides the Stokes modes, one finds a whole spectrum of additional unphysical modes, corresponding to those of the heat equation. Thus, the initial physical problem has fundamentally been changed. This approach has been applied to compute the full Stokes spectrum [1] by the first time. Due to the choice of a unit square domain, the authors were able to separate the Stokes modes from those belonging to the heat equation. An other strategy consists in applying a penalty method to solve the Stokes problem. In this case, the number of degrees of freedom still remains the same as those in the unconstrained Laplacian problem.

In Section 3, we focus on the two formulations considering only the velocity as variable: the penalty method and the divergence-free Galerkin approach. In the framework of spectral element approximation schemes, a stable spectral element is proposed for each method. For the penalty method, a COOL approach $[2,3]$ is made and the unphysical modes can be pushed towards $\lambda=0$. For the divergence-free Galerkin approach, two strategies christened "explicit" and "implicit" are detailed. The explicit strategy consists in using the properties of the kernel of the grad(div) operator to construct a divergencefree basis. Such a basis has the right number of degrees of freedom, thus delivering the exact number of Stokes eigenfunctions with high precision. The implicit strategy is a direct algebraic elimination process of the $\nabla \cdot \boldsymbol{u}=0$ constraint. This leads to a sparse matrix elimination process, described in detail in [2]. It delivers the right number of highly precise Stokes modes.

Finally, in Section 4, some numerical experiments are performed to prove the efficiency of the proposed methods and a comparison between the different approaches is given.

\section{The Stokes eigenvalue problem: continuous version}

Let $\Omega \subset I R^{d}, d=2,3$, be a Lipschitz domain, the generic point of $\Omega$ is denoted $x$. The symbol $L^{2}(\Omega)$ stands for the usual Lebesgue space and $H^{1}(\Omega)$, the Sobolev space that involves all the functions that are, together with their gradient, in $L^{2}(\Omega) \cdot \mathcal{C}(\Omega)$ denotes the space of continuous functions defined in $\Omega$.

The continuous Stokes eigenvalue problem reads: Find $a$ vector $\boldsymbol{u}$ and $\lambda^{2} \in I R^{+}$such 
that

$$
\begin{array}{ll}
-\Delta \boldsymbol{u}=\lambda^{2} \boldsymbol{u}, & \text { for } x \in \Omega, \\
\nabla \cdot \boldsymbol{u}=0, & \text { for } x \in \Omega, \\
\boldsymbol{u}=0, & \text { for } \boldsymbol{x} \in \partial \Omega,
\end{array}
$$

where $I R^{+}$denotes the set of positive real numbers, including zero. For the sake of simplicity we assume here that $\Omega$ is the reference domain $(-1,+1)^{2}$.

Problem (2.1) is often solved using different strategies that we briefly recall here after.

\section{Velocity-Pressure formulation}

$$
\begin{array}{ll}
-\Delta \boldsymbol{u}+\nabla p=\lambda^{2} \boldsymbol{u}, & \text { for } \boldsymbol{x} \in \Omega, \\
\nabla \cdot \boldsymbol{u}=0, & \text { for } \boldsymbol{x} \in \Omega, \\
\boldsymbol{u}=0, & \text { for } \boldsymbol{x} \in \partial \Omega .
\end{array}
$$

Its weak formulation writes: Find $\boldsymbol{u} \in\left(H_{0}^{1}(\Omega)\right)^{2}, p \in L_{0}^{2}(\Omega)$ and $\lambda^{2} \in I R^{+}$such that

$$
\begin{array}{ll}
\int_{\Omega} \nabla \boldsymbol{u} \cdot \nabla \boldsymbol{v} d \boldsymbol{x}-\int_{\Omega} p \nabla \cdot \boldsymbol{v} d \boldsymbol{x}=\lambda^{2} \int_{\Omega} \boldsymbol{u} \cdot \boldsymbol{v} d \boldsymbol{x}, & \forall \boldsymbol{v} \in\left(H_{0}^{1}(\Omega)\right)^{2}, \\
\int_{\Omega} \nabla \cdot \boldsymbol{u} q d \boldsymbol{x}=0, & \forall q \in L_{0}^{2}(\Omega) .
\end{array}
$$

Stream function approach We introduce a stream function $\psi \in H^{2}(\Omega)^{d}$ [1]. Problem (2.1) can be reformulated as:

$$
\begin{array}{ll}
\left(u_{x}, u_{y}\right)=\left(\frac{\partial \psi}{\partial y},-\frac{\partial \psi}{\partial x}\right), & \text { for } x \in \Omega, \\
\left(\lambda^{2}-\Delta\right) \Delta \psi=0, & \text { for } x \in \Omega, \\
\psi=\frac{\partial \psi}{\partial n}=0, & \text { for } x \in \partial \Omega .
\end{array}
$$

However, in this paper we prefer to focus on methods involving only $\boldsymbol{u}$ as unknown. The first one is called Penalty method.

Penalty method This method consists in taking into account the divergence free constraint by adding a term of penalty to control the level of divergence when solving the eigenvalue problem.

The penalty formulation, called also regularization method (see [8]), reads: Find $\boldsymbol{u} \in$ $\left(H_{0}^{1}(\Omega)\right)^{2}$ and $\lambda^{2} \in I R^{+}$such that

$$
-\Delta \boldsymbol{u}-\alpha \nabla(\nabla \cdot \boldsymbol{u})=\lambda^{2} \boldsymbol{u}, \quad \text { for } \boldsymbol{x} \in \Omega .
$$


Its variational formulation writes: Find $\boldsymbol{u} \in\left(H_{0}^{1}(\Omega)\right)^{2}$ and $\lambda^{2} \in I R^{+}$such that

$$
\int_{\Omega} \nabla \boldsymbol{u} \cdot \nabla \boldsymbol{v} d \boldsymbol{x}+\alpha \int_{\Omega} \nabla \cdot \boldsymbol{u} \nabla \cdot \boldsymbol{v} d \boldsymbol{x}=\lambda^{2} \int_{\Omega} \boldsymbol{u} \cdot \boldsymbol{v} d \mathbf{x}, \quad \forall \boldsymbol{v} \in\left(H_{0}^{1}(\Omega)\right)^{2} .
$$

In practice, the infinite dimensional problem (2.6) is replaced by a finite dimensional one using a stable spectral element taking into account the constraint by an adequate choice of $\alpha$ (see [3]).

Divergence-free Galerkin approach The second method is called "divergence-free Galerkin approach" and starts from the fact that the system (2.6) can reduce to: Find $\boldsymbol{u} \in \mathbf{X}$ and $\lambda^{2} \in I R^{+}$such that

$$
\mathcal{S}(\boldsymbol{u}, \boldsymbol{v}):=\int_{\Omega} \nabla \boldsymbol{u} \cdot \nabla \boldsymbol{v} d \boldsymbol{x}=\lambda^{2} \int_{\Omega} \boldsymbol{u} \cdot \boldsymbol{v} d \boldsymbol{x}, \quad \forall \boldsymbol{v} \in \mathbf{X},
$$

where $\mathbf{X}$ is in the space defined by

$$
\mathbf{X}=\left\{\boldsymbol{v} \in\left(H_{0}^{1}(\Omega)\right)^{2}, \text { such that } \nabla \cdot \boldsymbol{v}=0\right\} .
$$

Again the infinite dimensional problem (2.7) is replaced by a finite dimensional one using a stable spectral element that will be developed later in this paper.

\section{The Stokes eigenvalue problem: discrete version}

We firstly introduce some notations and reminders. Let $\Sigma_{G L L}=\left\{\left(\xi_{i}, \rho_{i}\right) ; 0 \leq i \leq p\right\}$ and $\Sigma_{G L}=\left\{\left(\zeta_{i}, \omega_{i}\right) ; 1 \leq i \leq p\right\}$ respectively denote the sets of Gauss-Lobatto-Legendre and Gauss-Legendre quadrature nodes and weights associated to polynomials of degree $p$. These quantities are such that on $\Lambda:=]-1,+1[$

$$
\begin{aligned}
& \forall \Phi \in I P_{2 p-1}(\Lambda), \quad \int_{-1}^{+1} \Phi(\xi) d \xi=\sum_{j=0}^{p} \Phi\left(\xi_{j}\right) \rho_{j}, \\
& \forall \Phi \in I P_{2 p-1}(\Lambda), \quad \int_{-1}^{+1} \Phi(\zeta) d \zeta=\sum_{j=1}^{p} \Phi\left(\zeta_{j}\right) \omega_{j},
\end{aligned}
$$

where $I P_{p}(\Lambda)$ denotes the space of polynomials with degree $\leq p$. We recall that the nodes $\xi_{i}(0 \leq i \leq p)$ are solution to $\left(1-x^{2}\right) L_{p}^{\prime}(x)=0$ where $L_{p}$ denotes the Legendre polynomial of degree $p$, whereas $\zeta_{i}(1 \leq i \leq p)$ are solution to $L_{p}(x)=0$ (see [4]).

The canonical polynomial interpolation basis $h_{i}(x) \in I P_{p}(\Lambda)$ built on $\Sigma_{G L L}$ is given by the relationships:

$$
h_{i}(x)=-\frac{1}{p(p+1)} \frac{1}{L_{p}\left(\xi_{i}\right)} \frac{\left(1-x^{2}\right) L_{p}^{\prime}(x)}{\left(x-\xi_{i}\right)}, \quad-1 \leq x \leq+1, \quad 0 \leq i \leq p,
$$


with the elementary cardinality property

$$
h_{i}\left(\xi_{j}\right)=\delta_{i j,} \quad 0 \leq i, j \leq p,
$$

where $\delta_{i j}$ is Kronecker's delta symbol.

We also introduce a new family of polynomials functions $g_{i}(x)$ associated to the canonical basis (3.3) through the relationships:

$$
g_{i}(x)=h_{i}(x)-\beta_{i} L_{p}(x), \quad 0 \leq i \leq p,
$$

where the constants $\beta_{i}$ are such that all $g_{i}(x) \in I P_{p-1}(]-1,+1[)[2,3]$. The functions $g_{i}(x)$ have the following properties:

1. Their moments up to order $(p-1)$ are equal to those of their corresponding element in the Gauss-Lobatto-Legendre canonical basis, i.e.: For $0 \leq i \leq p$,

$$
\int_{-1}^{+1}\left(g_{i}(x)-h_{i}(x)\right) x^{j} d x=0, \quad \forall j, \quad 0 \leq j \leq(p-1) .
$$

The difference $\left(g_{i}(x)-h_{i}(x)\right)$ being proportional to $L_{p}(x)$ is orthogonal to all polynomials of degree less or equal to $(p-1)$.

2. Interpolation of their corresponding element in the canonical basis at the GaussLegendre nodes, i.e.: For $0 \leq i \leq p$,

$$
g_{i}\left(\zeta_{j}\right)=h_{i}\left(\zeta_{j}\right), \quad \forall j, \quad 1 \leq j \leq p .
$$

3. The constants $\beta_{i}$ can be obtained through a series expansion of (3.3) and one gets:

$$
\beta_{i}=\frac{1}{(p+1) L_{p}\left(\xi_{i}\right)}, \quad 0 \leq i \leq p .
$$

In [3] one can read more informations concerning these polynomial functions.

\subsection{Penalty method: SEM approximation}

The discrete version of problem (2.6) writes: Find $\boldsymbol{u}_{p} \in \boldsymbol{Y}_{p}$ and $\lambda^{2} \in I R^{+}$such that

$$
\mathcal{A}_{p}\left(\boldsymbol{u}_{p}, \boldsymbol{v}_{p}\right)+\alpha \mathcal{B}_{p}\left(\boldsymbol{u}_{p}, \boldsymbol{v}_{p}\right)=\lambda^{2}\left(\boldsymbol{u}_{p}, \boldsymbol{v}_{p}\right)_{p}, \quad \forall \boldsymbol{v}_{p} \in \boldsymbol{Y}_{p},
$$

where:

$$
\begin{aligned}
& \mathcal{A}_{p}\left(\boldsymbol{u}_{p}, \boldsymbol{v}_{p}\right)=\left(\nabla \boldsymbol{u}_{p}, \nabla \boldsymbol{v}_{p}\right)_{p}, \\
& \mathcal{B}_{p}\left(\boldsymbol{u}_{p}, \boldsymbol{v}_{p}\right)=\left(\nabla \cdot \boldsymbol{u}_{p}, \nabla \cdot \boldsymbol{v}_{p}\right)_{p} .
\end{aligned}
$$


Here $(\cdot, \cdots)_{p}$ is discrete scalar product based on Gauss Lobatto quadrature formula. $\boldsymbol{Y}_{p}$ is the space of polynomial functions of degree lower or equal to $p$ vanishing on $\partial \Omega$. It is assumed to ensure a stable approximation for $\operatorname{grad}(\mathrm{div})$ operator to avoid the phenomenon of spurious pollution [3]. Since $\boldsymbol{u}_{p}$ is equal to zero on the boundary, the solution $\boldsymbol{u}_{p} \in \mathbf{Y}_{\mathbf{p}}$ is approximated by $u_{r p}^{(0)}(x, y), u_{r p}^{(1)}(x, y)$ or $u_{r p}^{(2)}(x, y)$ according to the functional dependence and the regularity required $(r=x$ or $y)$.

$$
\begin{aligned}
& u_{r p}^{(0)}(x, y)=\sum_{i=1}^{p-1} \sum_{j=1}^{p-1} u^{r p}\left(\xi_{i}, \xi_{j}\right) g_{i}(x) g_{j}(y), \\
& u_{r p}^{(1)}(x, y)=\sum_{i=1}^{p} \sum_{j=1}^{p-1} u^{r p}\left(\xi_{i}, \xi_{j}\right) h_{i}(x) g_{j}(y), \\
& u_{r p}^{(2)}(x, y)=\sum_{i=1}^{p} \sum_{j=1}^{p-1} u^{r p}\left(\xi_{i}, \xi_{j}\right) g_{i}(x) h_{j}(y) .
\end{aligned}
$$

The superscript ${ }^{(1)}$ is used to represent quantities derived in direction $x$ while superscript (2) is used to represent quantities derived in direction $y$. The coefficients in the previous three expansions are the same thanks to (3.7).

Replacing $\boldsymbol{u}_{p}$ by the previous development in (3.9), the penalty discrete form writes

$$
\begin{aligned}
& \left(\partial_{x} u_{x p}^{(1)}, \partial_{x} v_{x p}^{(1)}\right)_{p}+\left(\partial_{y} u_{y p}^{(2)}, \partial_{y} v_{y p}^{(2)}\right)_{p}+\alpha\left(\partial_{x} u_{x p}^{(1)}+\partial_{y} u_{y p}^{(2)}, \partial_{x} v_{x p}^{(1)}+\partial_{y} v_{y p}^{(2)}\right)_{p} \\
= & \lambda^{2}\left(\boldsymbol{u}_{p}^{(0)}, \boldsymbol{v}_{p}^{(0)}\right)_{p}, \quad \forall \boldsymbol{v}_{p} \in \boldsymbol{Y}_{p} .
\end{aligned}
$$

\subsection{Divergence-free Galerkin approach}

The keystone of the divergence-free Galerkin approach is the construction of a discrete version $\boldsymbol{X}_{p}$ of the divergence free space $\boldsymbol{X}$ defined in equation (2.8).

According to $[2,5]$, we need the divergence to be a polynomial of degree less or equal to $p-1$. Consequently, we want to build a space:

$$
\mathbf{X}_{\mathbf{p}}=\left\{\boldsymbol{u}_{p} \in\left(I P_{p}(\Omega)\right)^{2} \mid \nabla \cdot \boldsymbol{u}_{p} \in I P_{p-1}(\Omega)\right\} \cap \mathbf{X} .
$$

Expanding $\boldsymbol{u}_{p}$ according to (3.12b) and (3.12c) its divergence is a polynomials of degree $p-1$. Consequently, if the divergence is orthogonal to all polynomial of $I P_{p-1}(\Omega)$, it is necessarily equal to 0 . This point gives a new characterization for $\mathbf{X}_{\mathbf{p}}$ :

$$
\mathbf{X}_{\mathbf{p}}=\left\{\boldsymbol{u}_{p} \in\left(I P_{p}^{0}(\Omega)\right)^{2} \mid \int_{\Omega}\left(\frac{\partial u_{x p}^{(1)}}{\partial x}+\frac{\partial u_{y p}^{(2)}}{\partial y}\right) q d \mathbf{x}=0, \quad \forall q \in I P_{p-1}(\Omega)\right\} .
$$

We want to build a basis of $\mathbf{X}_{\mathbf{p}}$. The first step consists in determining the size of this space.

$$
\operatorname{dim} \boldsymbol{X}_{\mathbf{p}}=\operatorname{dim}\left(I P_{p}^{0}(\Omega)\right)^{2}-p_{2},
$$


where $p_{2}$ is the number of necessary and sufficient equations to ensure $\nabla \cdot \boldsymbol{u}_{p} \equiv 0$. $\nabla \cdot \boldsymbol{u}_{p} \in I P_{p-1}(\Omega)$ therefore $p_{2} \leq p^{2}$.

There are 2 dependent equations in $2 D$ (see [6] for details) since:

$$
\begin{array}{ll}
\int_{\Omega} \nabla \cdot \boldsymbol{u}_{p} L_{0}(x) L_{0}(y) d \mathbf{x}=0, & \forall \boldsymbol{u}_{p} \in\left(I P_{p}^{0}(\Omega)\right)^{2}, \\
\int_{\Omega} \nabla \cdot \boldsymbol{u}_{p} L_{p}^{\prime}(x) L_{p}^{\prime}(y) d \mathbf{x}=0, & \forall \boldsymbol{u}_{p} \in\left(I P_{p}^{0}(\Omega)\right)^{2} .
\end{array}
$$

Polynomials $L_{0}(x) L_{0}(y)$ and $L_{p}^{\prime}(x) L_{p}^{\prime}(y)$ are spurious modes and reduce the number of independent equations from $p^{2}$ to $p^{2}-2$. Consequently, we require $p_{2}=p^{2}-2$ test functions $q$ to ensure $\int_{\Omega} \nabla \cdot \boldsymbol{u}_{p} q d \mathbf{x}=0$.

$$
\operatorname{dim} \mathbf{X}_{\mathbf{p}}=\operatorname{dim}\left(I P_{p}^{0}(\Omega)\right)^{2}-p_{2}=2(p-1)^{2}-\left(p^{2}-2\right)=(p-2)^{2} .
$$

After the computation of the size of $\mathbf{X}_{\mathbf{p}}$ (denoted $p_{1}=(p-2)^{2}$ in the sequel), we propose two strategies to compute a divergence-free basis.

\subsubsection{Divergence-free Galerkin explicit approach}

We consider the following eigenvalue problem:

$$
\begin{array}{ll}
-\nabla(\nabla \cdot \boldsymbol{u})=\lambda^{2} \boldsymbol{u}, & \text { for } \boldsymbol{x} \in \Omega, \\
\boldsymbol{u}=0, & \text { for } \boldsymbol{x} \in \partial \Omega .
\end{array}
$$

The kernel of the $\operatorname{grad}(\mathrm{div})$ operator includes all the modes $\boldsymbol{u}_{s, p}^{k}$ associated to $\lambda^{2}=0$ and $\nabla \cdot \boldsymbol{u}_{s, p}^{k}=0$. It constitutes a basis for the subspace $\mathbf{X}_{p}$. Its size is $(p-2)^{2}$ and then $\boldsymbol{u}_{p} \in \mathbf{X}_{\mathbf{p}}$ can be decomposed according to the following form:

$$
\boldsymbol{u}_{p}=\sum_{k=1}^{(p-2)^{2}} \beta_{k} \boldsymbol{u}_{s, p}^{k}
$$

Replacing $\boldsymbol{u}_{p}$ by the previous development in (2.7), the discrete variational formulation writes: Find $\boldsymbol{u}_{p} \in \mathbf{X}_{\mathbf{p}}$ and $\lambda^{2} \in I R^{+*}$ such that

$$
\sum_{k=1}^{(p-2)^{2}}\left(\nabla \boldsymbol{u}_{s, p}^{k}, \nabla \boldsymbol{u}_{s, p}^{i}\right)_{p} \beta_{k}=\lambda^{2} \sum_{k=1}^{(p-2)^{2}}\left(\boldsymbol{u}_{s, p}^{k}, \boldsymbol{u}_{s, p}^{i}\right)_{p} \beta_{k}, \forall \boldsymbol{u}_{s, p}^{i} \in \mathbf{X}_{\mathbf{p}} .
$$

This can be written:

$$
\underline{\mathcal{S}}^{e} \underline{\beta}=\lambda^{2} \underline{\mathcal{M}}^{e} \underline{\beta} .
$$

The stiff matrix $\mathcal{S}^{e}$ and mass matrix $\mathcal{M}^{e}$ are symmetric and positive definite and are defined by:

$$
\underline{\mathcal{S}}_{i k}^{e}=\left(\nabla \boldsymbol{u}_{s, p}^{k}, \nabla \boldsymbol{u}_{s, p}^{i}\right)_{p}, \quad \underline{\mathcal{M}}_{i k}^{e}=\left(\boldsymbol{u}_{s, p}^{k}, \boldsymbol{u}_{s, p}^{i}\right)_{p},
$$

for $\left(1 \leq i, k \leq(p-2)^{2}\right)$. 


\subsubsection{Divergence-free Galerkin implicit approach}

As highlighted before, the main difficulty of the problem (2.1) consists in satisfying the incompressibility constraint $\nabla \cdot \boldsymbol{u}=0$. Classical approaches usually satisfy operator equations strongly with as many equations as degrees of freedom for the velocity while incompressibility constraint is only satisfied weakly with fewer equations than degrees of freedom for the divergence. Contrary to the classical approaches, our objective is to favor the incompressibility constraint in comparison with the other equations. Our strategy, firstly introduced in [2], consists in sharing the degrees of freedom of $u$ in a relevant way to satisfy:

- The incompressibility constraint in strong sense.

- The other equations in weak sense.

Let $\boldsymbol{u}_{p}$ be in $\boldsymbol{X}_{\mathbf{p}}$. The divergence of $\boldsymbol{u}_{p}$ is orthogonal to $p^{2}-2$ polynomials of degree $p-1$. It is equivalent to saying that the divergence of $\boldsymbol{u}_{p} \in \mathbf{X}_{\mathbf{p}}$ nullifies in $p^{2}-2$ Gauss points. The algebraic divergence equation writes $D \boldsymbol{u}_{p}=0$ (see Figure (1)).

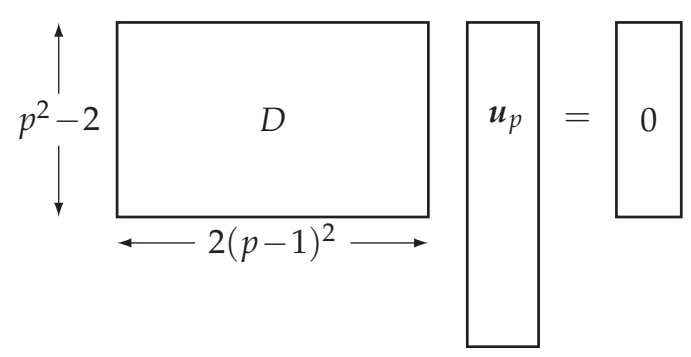

Figure 1: Algebraic system.

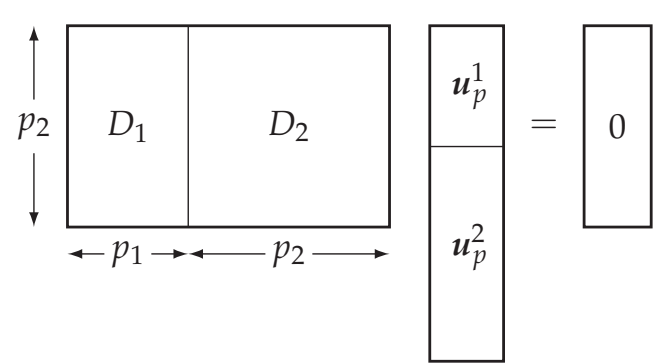

Figure 2: Decomposition of D.

$\mathrm{D}$ is a rectangular matrix with $p_{2}=p^{2}-2$ rows and $2(p-1)^{2}$ columns.

Then, one splits $D$ into $D_{1} \oplus D_{2}$ and $\boldsymbol{u}_{p}$ into $\boldsymbol{u}_{1 p} \oplus \boldsymbol{u}_{2 p}$ (see Figure (2)).

Since, the $p^{2}-2$ lines of $\mathrm{D}$ are independent, there is at least one choice of matrix $D_{2}$ invertible. Equation $D \boldsymbol{u}_{p}=0$ becomes:

$$
D_{1} \boldsymbol{u}_{1 p}+D_{2} \boldsymbol{u}_{2 p}=0 .
$$

For instance, $\boldsymbol{u}_{1}$ contains the $p_{1}$ first values of $\boldsymbol{u}_{p}$ and consequently $\boldsymbol{u}_{2 p}$ contains the $p_{2}$ remaining values. Figure 3 displays the sizes of the matrices $D_{2}$ and $D_{1}$.

Since $D_{2}$ is invertible, the system leads to a relation between $\boldsymbol{u}_{1 p}$ and $\boldsymbol{u}_{2 p}$ :

$$
\boldsymbol{u}_{2 p}=-D_{2}^{-1} D_{1} \boldsymbol{u}_{1 p} .
$$

Eq. (3.15) is crucial since it means that if we have any part $\boldsymbol{u}_{1 p}$ of $\boldsymbol{u}_{p}$, we can build the complementary $\boldsymbol{u}_{2 p}$ such that divergence of $\boldsymbol{u}_{p}$ equals 0 . This argument allows us to build a basis of $\mathbf{X}_{\mathbf{p}}$. 


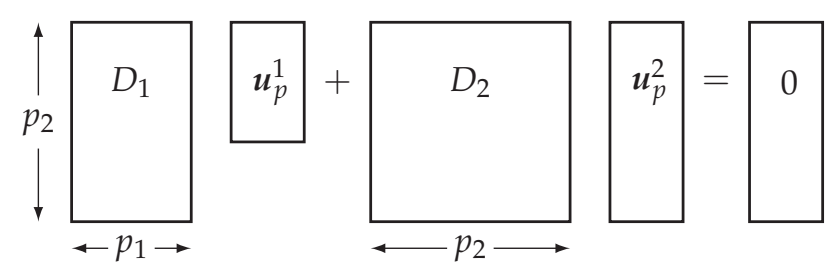

Figure 3: Algebraic system $D_{1} u_{1 p}+D_{2} u_{2 p}=0$.

We consider $\boldsymbol{v}_{p} \in\left(I P_{p}^{0}(\Omega)\right)^{2}$. Our strategy consists in combining implicitly:

- A reduction from $v_{p}$ to $v_{1 p}$,

- An extension from $\boldsymbol{v}_{1 p}$ to $\boldsymbol{w}_{p}=\left(\boldsymbol{v}_{1 p}, \boldsymbol{v}_{2 p}\right)$ such that $\nabla \cdot \boldsymbol{w}_{p}=0$ ensured by the multiplication of $v_{1 p}$ by the matrix

$$
M=\left[\begin{array}{c}
I_{p_{1}} \\
-D_{2}^{-1} D_{1}
\end{array}\right]
$$

The matrix $M$ is a two blocks matrix. The first block is a matrix of order $p_{1}$ equal to identity. The second block contains $p_{2}$ rows and $p_{1}$ columns. It ensures the passage from $\boldsymbol{v}_{1 p}$ to $\boldsymbol{v}_{2 p}$.

- For each $\boldsymbol{v}_{p} \in\left(I P_{p}^{0}(\Omega)\right)^{2}$, one associates a vector $\boldsymbol{w}_{p}$ of $\mathbf{X}_{\mathbf{p}}$.

By consequent, our strategy for the construction of a basis of $\mathbf{X}_{\mathbf{p}}$ consists in:

- Choosing $p_{1}=(p-2)^{2}$ vectors $\left(\boldsymbol{v}_{p}^{k}\right)_{k=1, \ldots, p_{1}}$ of the basis of $\left(I P_{p}^{0}(\Omega)\right)^{2}$ (for instance, the $(p-2)^{2}$ first vectors),

- For each one of these $p_{1}$ vectors, we consider its $p_{1}$-size reduced part denoted $v_{1 p^{\prime}}^{k}$

- We carry out the divergence-free extension $\left(\boldsymbol{w}_{p}^{k}\right)_{k=1, \ldots, p_{1}}=\left(M \boldsymbol{v}_{1 p}^{k}\right)_{k=1, \ldots, p_{1}}$.

The $\left(\boldsymbol{w}_{p}^{k}\right)_{k=1, \ldots, p_{1}}$ family is a basis of $\mathbf{X}_{\mathbf{p}}$ and every $\boldsymbol{u}_{p} \in \mathbf{X}_{\mathbf{p}}$ can be decomposed according to the following form:

$$
\boldsymbol{u}_{p}=\sum_{k=1}^{p_{1}} \gamma_{k} \boldsymbol{w}_{p}^{k}
$$

Replacing $\boldsymbol{u}_{p}$ by the previous development, the discrete variational formulation writes: Find $\boldsymbol{u}_{p} \in \mathbf{X}_{\mathbf{p}}$ and $\lambda^{2} \in I R^{+*}$ such that

$$
\sum_{k=1}^{p_{1}}\left(\nabla \boldsymbol{w}_{p}^{k}, \nabla \boldsymbol{w}_{p}^{\mathrm{i}}\right)_{p} \gamma_{k}=\lambda^{2} \sum_{k=1}^{p_{1}}\left(\boldsymbol{w}_{p}^{k}, \boldsymbol{w}_{p}^{\mathrm{i}}\right)_{p} \gamma_{k}, \forall \boldsymbol{w}_{p}^{\mathrm{i}} \in \mathbf{X}_{\mathbf{p}}
$$


Table 1: Maximum and minimum of the $L^{2}(\Omega)$-norm of the divergence of all the Stokes eigenmodes as a function of $p$.

\begin{tabular}{||c|ccccc||}
\hline$p$ & 4 & 8 & 12 & 16 & 20 \\
\hline $\min \left\|\nabla \cdot \boldsymbol{u}_{p}\right\|_{L^{2}(\Omega)}$ & $8.1 \times 10^{-7}$ & $8.97 \times 10^{-7}$ & $8.97 \times 10^{-7}$ & $8.97 \times 10^{-7}$ & $8.97 \times 10^{-7}$ \\
$\max \left\|\nabla \cdot \boldsymbol{u}_{p}\right\|_{L^{2}(\Omega)}$ & $8.92 \times 10^{-6}$ & $7.66 \times 10^{-5}$ & $2.54 \times 10^{-5}$ & $5.95 \times 10^{-4}$ & $1.15 \times 10^{-3}$ \\
\hline
\end{tabular}

This can be written:

$$
\underline{\mathcal{S}}^{i} \underline{\gamma}=\lambda^{2} \underline{\mathcal{M}}^{i} \underline{\gamma}
$$

with for $\left(1 \leq i, k \leq p_{1}\right)$,

$$
\underline{\mathcal{S}}_{i k}^{i}=\left(\nabla \boldsymbol{w}_{p}^{k}, \nabla \boldsymbol{w}_{p}^{\mathrm{i}}\right)_{p}, \quad \underline{\mathcal{M}}_{i k}^{i}=\left(\boldsymbol{w}_{p}^{k}, \boldsymbol{w}_{p}^{\mathrm{i}}\right)_{p} .
$$

$\underline{\mathcal{S}}^{i}$ and $\underline{\mathcal{M}}^{i}$ refer respectively to the Laplace operator and mass matrices expressed on the basis $\boldsymbol{w}_{p}$.

Finally, this system is equivalent to:

$$
M^{T} A M \boldsymbol{u}_{p 1}=\lambda^{2} M^{T} B M \boldsymbol{u}_{p 1}
$$

where $A$ and $B$ refer respectively to the classical Laplacian and mass matrices.

\section{Numerical results}

This section discusses some numerical results. We will apply each of the three approaches to compute the Stokes eigenvalues and associated eigenfunctions.

Penalty approach Figure 4 displays the convergence for the lowest eigenvalue of problem (2.5) for several values of $\alpha$.

One can see that the choice of $\alpha$ leads to slightly different convergence behaviors. For double precision arithmetic, $\alpha=10^{7}$ appears to give the best convergence results. With an increasing polynomial degree to represent the eigenfunction, the eigenvalue converges exponentially as expected for $p \leq 9$. Increasing $p$ further does not improve the accuracy of the eigenvalue, with the precision limited to $10^{-6}$. Table 1 shows the limit in precision for the incompressibility condition for $\alpha=10^{7}$ as a function of $p$.

The eigenvalue problem (3.9) gives $2(p-1)^{2}$ eigenvalues and associated eigenvectors corresponding to the degrees of freedom in $\boldsymbol{Y}_{p}$. Among these eigenvalues, there are the Stokes eigenvalues and the non-zero eigenvalues of the grad(div) operator multiplied by $\alpha$. The number of Stokes eigenvalues $\mathcal{N}_{S}$ corresponds to the size of the kernel 


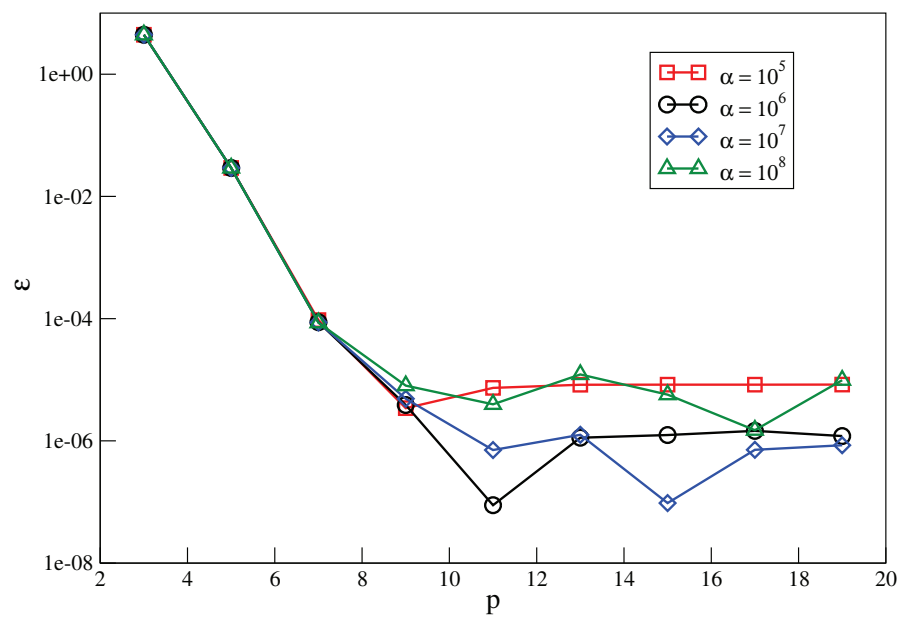

Figure 4: Convergence plots obtained using the penalty method for the first Stokes mode $\left(\lambda^{2}=13.086172791\right)$ as a function of the polynomial order $p$ for several values of $\alpha$.

of the discretized grad(div) operator, i.e. to the number of zero eigenvalues. As said in Section (3.2.1), it can be proved that this number is equal to $(p-2)^{2}$. Consequently, the resolution of the problem (3.9) leads to $\mathcal{N}_{S}=(p-2)^{2}$ Stokes eigenmodes. The $p^{2}-2$ remaining eigenmodes are those of the class of non-zero eigenvalues of the grad(div) operator multiplied by $\alpha$.

Figure 5 illustrates the convergence of the difference $\epsilon$ between the four lowest Stokes eigenvalues as a function of $p$ computed by our method with those produced in [1] for $\alpha=10^{7}$ on a semi-logarithmic scale. The error is exponentially decreasing as expected for $p \leq 11$ and then stagnates.

Divergence-free Galerkin explicit approach Figure 6 illustrates the convergence of the difference $\epsilon$ between the four lowest Stokes eigenvalues as a function of $p$ computed by the divergence-free Galerkin explicit approach with those produced in [1] on a semilogarithmic scale. The error is exponentially decreasing as expected.

Divergence-free Galerkin implicit approach To validate our divergence-free Galerkin implicit approach, we have computed the Stokes eigenvalues and compared with those obtained in [1]. Figure 7 shows the convergence for the four lowest eigenvalues as a function of $p$ on a semi-logarithmic scale. The calculation of the eigenvalues converges exponentially as expected.

It has been shown theoretically that the eigenmodes have a global structure with an infinite series of Moffat corner vortices [7] of increasingly smaller amplitude. The right part of the Figure 8 represents the $u_{p x}$ component of the thirteenth eigenmode. The amplitude is 0.852 . In the center of the figure, we can see the first Moffat vortex in the left 


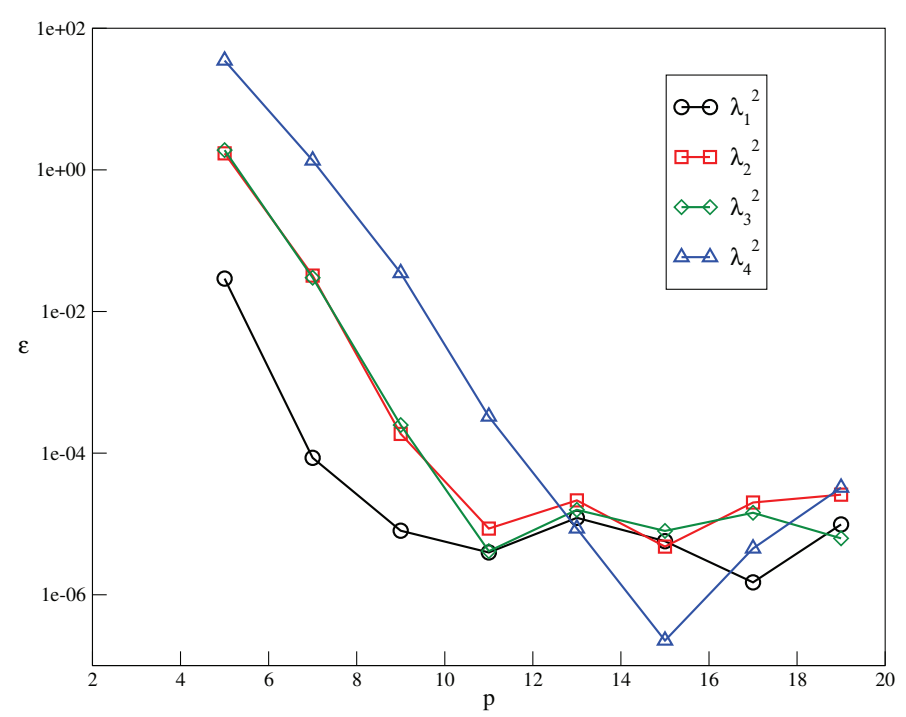

Figure 5: Convergence plots obtained using the penalty method for the four lowest divergence-free modes as a function of $p$. Again, $\alpha=10^{7}$.

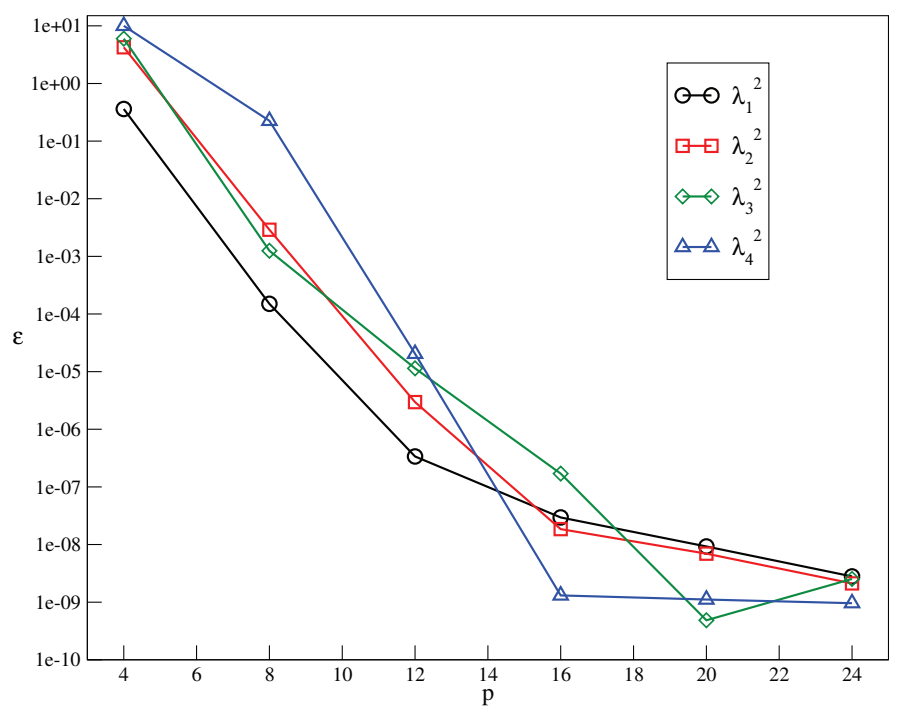

Figure 6: Convergence plots obtained using the divergence-free Galerkin explicit approach method for the four lowest divergence-free modes as a function of $p$.

upper corner of the geometry with an amplitude of $1 \times 10^{-3}$. At last, in the left side of the figure, the second Moffat vortex has an amplitude of $2 \times 10^{-6}$. These results are in accordance with the theoretical ones.

Comparison between the different approaches The three strategies described in this paper give the expected number of Stokes eigenvalues with a high precision. Nonethe- 


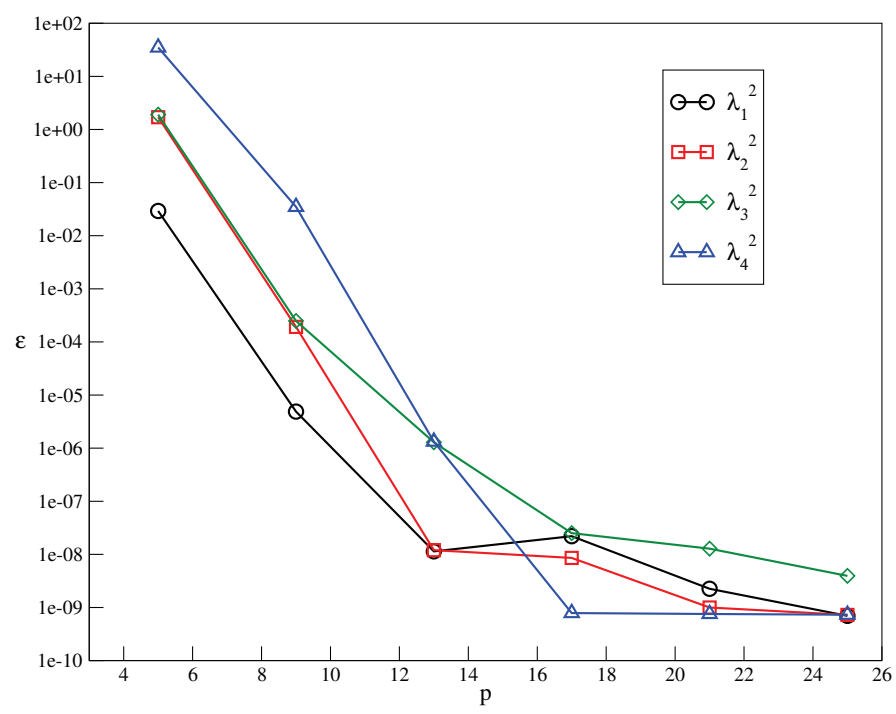

Figure 7: Relative error $\epsilon$ for the for the four lowest Stokes eigenvalues as a function of $p$ on a semi-logarithmic scale with the divergence-free Galerkin implicit approach.

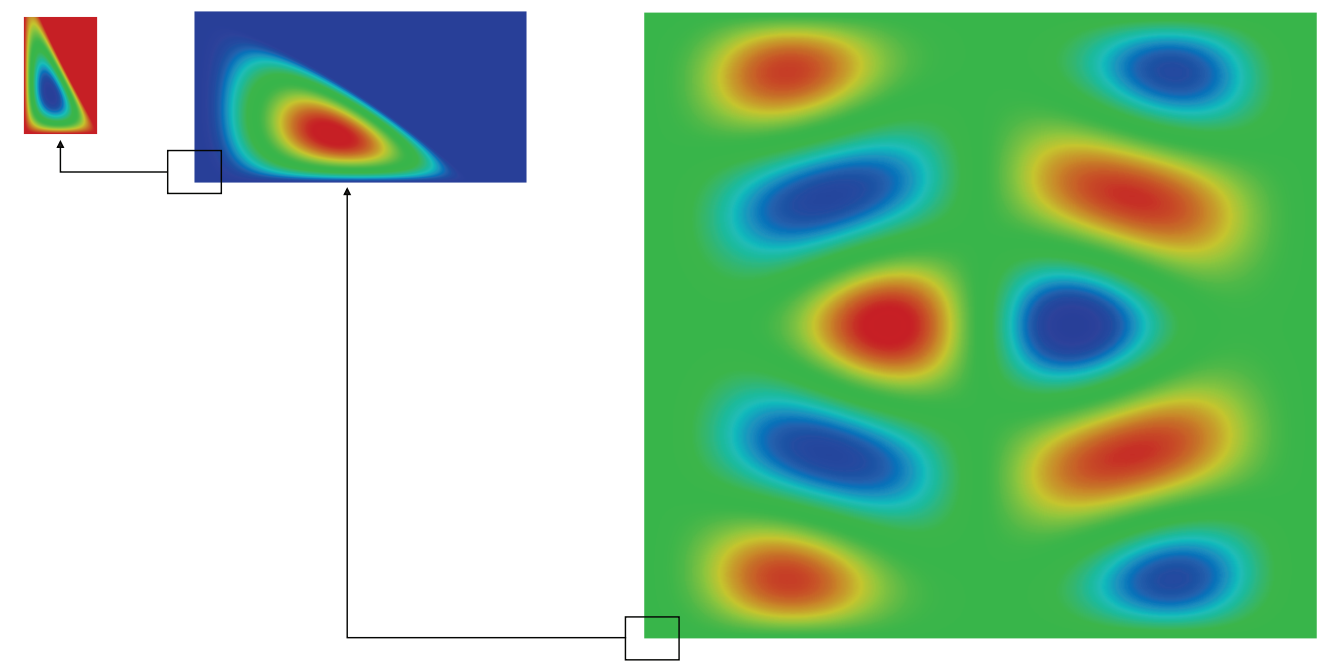

Figure 8: $u_{p x}$ component of $13^{\text {th }}$ Stokes eigenvector: Moffatt vortices in the corners.

less, Figure 5 compared with Figure 6 and Figure 7 indicates that the penalty approach is less accurate that the two other ones. Moreover, the divergence of the eigenvectors computed by the penalty approach is not identically null and its level can depend on the tricky choice of the values of $\alpha$. On the contrary, the divergence-free Galerkin approaches computed eigenvectors that are perfectly divergence-free. As drawback of the explicit version, we can mention that an initial computation of the kernel of the discretized $\operatorname{grad}($ div) operator has to be performed leading to the resolution of two eigenvalue prob- 
lems. Consequently, due to the reasons mentioned above, in our opinion, among the three strategies studied in this paper, the best one is the divergence-free Galerkin implicit approach.

\section{References}

[1] E. Leriche and G. Labrosse, Stokes eigenmodes in a square domain and the stream functionvelocity correlation, Journal of Computational Physics, 200 (2004), 489-511.

[2] E. Ahusborde, R. Gruber, M. Azaïez and M.L. Sawley, Physics-conforming constraintsoriented numerical method, Physical Review E, 75 (2007), 056704.

[3] E. Ahusborde, M. Azaïez and R. Gruber, Constraint oriented spectral element method, Lecture Notes in Computational Science and Engineering, 76 (2011), 93-100.

[4] M.O. Deville, P.F. Fischer, and E.H. Mund, High-order methods for incompressible fluid flow, Cambridge University Press, Cambridge, 2002.

[5] E. Ahusborde, High order method for the -grad(div) operator and applications, $\mathrm{PhD}$ thesis, University of Bordeaux, 2007.

[6] C. Bernardi and Y. Maday, Spectral methods in handbook of numerical analysis, P.G. Ciarlet and J.L. Lions, North-Holland, 1997.

[7] H. K. Moffatt, Viscous and resistive eddies near a sharp corner, Journal of Fluid Mechanics, 18 (1964), 1-18.

[8] V. Girault and P. Raviart, Finite element methods for Navier-Stokes equations, Series in Computational Mathematics, Springer-Verlag, Berlin, 1986.

[9] R. Gruber and J. Rappaz, Finite element methods in linear ideal MHD, Springer Series in Computational Physics, Springer-Verlag, Berlin, 1985. 